Revue d'histoire de l'Amérique française

Q REVUE D.HISTOIRE DE L'AMÉRIQUE FRANÇAISE

\title{
Deux piliers nous ont quittés
}

Juliette Lalonde-Rémillard (1916-2014). Lise McNicoll

(1950-2015)

Volume 69, numéro 1-2, été-automne 2015

URI : https://id.erudit.org/iderudit/1034586ar

DOI : https://doi.org/10.7202/1034586ar

Aller au sommaire du numéro

Éditeur(s)

Institut d'histoire de l'Amérique française

ISSN

0035-2357 (imprimé)

1492-1383 (numérique)

Découvrir la revue

Citer ce document

(2015). Deux piliers nous ont quittés : Juliette Lalonde-Rémillard (1916-2014).

Lise McNicoll (1950-2015). Revue d'histoire de l'Amérique française, 69(1-2),

7-10. https://doi.org/10.7202/1034586ar d'utilisation que vous pouvez consulter en ligne.

https://apropos.erudit.org/fr/usagers/politique-dutilisation/ 


\title{
Deux piliers nous ont quittés Juliette Lalonde-Rémillard (I9I6-20I4) \\ Lise McNicoll (I950-2015)
}

\begin{abstract}
Coup sur coup, à six mois de distance, les deux figures les plus présentes à l'Institut et à la Revue d'histoire de l'Amérique française nous ont quittés: celles qui furent leurs valeureuses secrétaires, depuis le début en 1946 jusqu'en 2010, soit plus de 60 ans !
\end{abstract}

\section{JULIETTE LALONDE-RÉMILLARD}

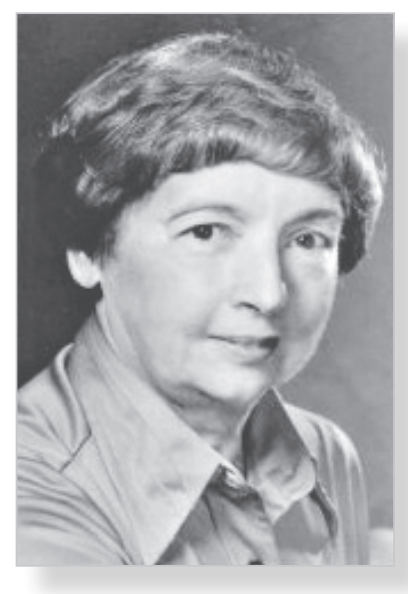

Source: La Fondation LionelGroulx
Juliette Lalonde était la nièce de Lionel Groulx. Il lui a proposé de devenir sa secrétaire le jour de ses 21 ans, le 19 septembre 1937. Elle l'a accompagné durant toute sa vie, y compris au 261, avenue Bloomfield, à Outremont, à partir de 1939. Elle sera la secrétaire de la Fondation Lionel-Groulx, depuis le début en 1956 jusqu'à sa retraite en 1999 , à l'âge de 83 ans. Elle fut la cheville ouvrière de la publication des Mémoires de Groulx (1970-1974) et de l'édition critique de ses inédits, notamment de sa Correspondance, dont quatre tomes sont parus (1989-2013). Le tome 4 contient d'ailleurs, de la plume de sa collaboratrice 
Giselle Huot, un remarquable «Hommage à Juliette Lalonde-Rémillard», qu'on peut facilement consulter sur Internet.

Madame Rémillard, nom sous lequel nous l'avons connue pendant plusieurs années, a publié beaucoup de ses souvenirs, attachants comme elle pouvait l'être elle-même. De sa conférence «Lionel Groulx, l'homme que j'ai connu " (Fides, 2000), tirons ces quelques lignes sur les débuts de l'IHAF en 1946 et de la RHAF en 1947 :

[...] à l'âge où d'autres prennent leur retraite, Groulx fondait, en 1946, un Institut d'histoire de l'Amérique française. [...] Il m'expose son projet avec entrain. Il me demande tout simplement si je crois pouvoir lui apporter mon aide pour ce surcroît de travail. J'accepte d'emblée.

Parlant ensuite des débuts de la Revue:

Au soir de la fondation, la caisse ne connaît encore que le vide absolu. Pas le moindre sou. [...] Nous menions de front la chasse aux annonces, aux contrats d'imprimerie, aux abonnés, la chasse aux articles, nous assumions la rédaction, la correction des épreuves, même l'expédition. C'était un véritable défi, mais nous avons réussi (p. 43-44).

La correction d'épreuves! Elle en était, comme l'écrit si bien Giselle Huot, «la championne toutes catégories». Relisons Groulx, dans le tome 3 de ses Mémoires:

Avec la fondation de l'Institut d'histoire de l'Amérique française et de sa revue, Juliette, déjà rompue à ce métier, s'instituera correctrice d'épreuves; elle se chargera de toute l'administration de l'Institut. Aujourd'hui, en 1959, voilà donc vingt-deux ans que la brunette aux yeux noirs est à mon service. Elle aura été ma collaboratrice, mêlée à toutes mes œuvres, à toute ma vie. [...]

Vive, enjouée, le rire facile, [...] résistante au travail, elle s'est donnée à sa tâche avec le dévouement entier, cordial, joyeux, qui, semble-t-il, n’appartient qu'aux femmes. (p. 357).

Vocabulaire d’une époque. Les années qui suivront, Juliette Rémillard poursuivra ce précieux travail avec les présidents et présidentes de l'IHAF et les directeurs et directrices de la RHAF, un monde où les femmes tiennent désormais une place plus déterminante.

Et que dire de son travail au Centre de recherche Lionel-Groulx, fondé en 1976? J'emprunte à Juliette elle-même la description des principaux buts de ce centre: "Constituer un fonds d'archives, créer un climat de travail fécond, d'initiative, faire en sorte que le chercheur ou l'étudiant puisse travailler avec aisance et facilité» (p. 47). Sa recherche pour les fonds 
d'archives des principales figures nationalistes du Canada français a été déterminante, de même que son accueil des chercheurs, si apprécié.

\section{LISE MCNICOLL}

C'est dans ce monde qu'apparaît Lise McNicoll, engagée à plein temps à l'Institut en avril 1978. Elle remplaçait Juliette Rémillard, qui se concentrait dès lors sur la Fondation et l'édition critique de Groulx. Comment enfiler d'aussi grandes chaussures? Avec beaucoup de détermination et de savoir-faire, Lise se mit à l'œuvre. Elle partageait avec madame Rémillard ce même culte du travail bien fait et un dévouement entier à l'Institut et à la Revue.

Avec les moyens électroniques qui

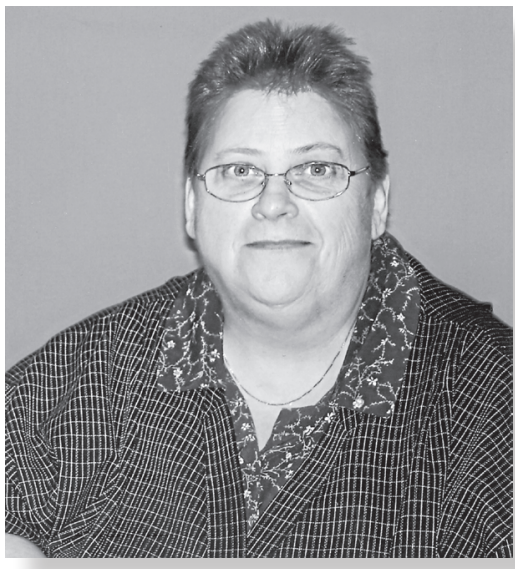

Source: La Fondation Lionel-Groulx sont aujourd'hui les nôtres, on ne se souvient guère de ce que pouvaient représenter la mise en pages et la correction des six cents pages et plus que publie chaque année la Revue. Sans oublier les relations avec les auteurs d'articles ou de comptes rendus, surtout les retardataires..., avec les imprimeurs ou l'administration des abonnements. L'Institut représentait lui aussi une tâche aux mille exigences: recherche de subventions, aux formulaires toujours plus longs et compliqués au fil des ans, organisation des congrès.

Le congrès annuel: voilà un champ d'action où Lise a fait merveille pendant de nombreuses années. Les organisateurs de ces congrès se souviennent de la chance qu' ils ont eue de pouvoir compter sur elle, qui savait comment faire tourner la machine, était toujours là en soutien, s'assurait que rien d'important ne soit oublié, de la gestion des inscriptions à la surveillance du budget. Elle était toujours prompte à accueillir la relève, les jeunes, qui l'apprécièrent beaucoup.

Et puis, ces changements réguliers de direction: les présidents, présidentes de l'Institut, directeurs, directrices de la RHAF se succédaient à peu près aux trois ans, avec chacun, chacune, sa personnalité, ses manières de faire. Lise savait s'adapter à chacun, mais on conçoit que sa situation ne devait pas être facile tous les jours. Le sourire et la bonne humeur n'en étaient pas moins au rendez-vous, même quand sa santé commença à lui 
poser de sérieux problèmes qui, finalement, en 2010, lui firent prendre sa retraite, en même temps que l'Institut devait déménager du 261, avenue Bloomfield, à l'Université de Montréal. On lui rendit un hommage bien mérité lors du congrès de 2011.

Terminons par deux témoignages de président.es de l'Institut, tirés du Bulletin, le premier lors du cinquantenaire de la revue et le second lors de retraite de Lise. Leur convergence ne manque pas de nous frapper:

Je tiens à remercier surtout Lise McNicoll, mémoire vivante et chaleureuse des vingt dernières années; point d'ancrage indispensable pour un exécutif écartelé entre quatre villes différentes; point d'encrage efficace dans toutes nos écritures (Micheline Dumont, 1997).

Je souhaite $[\ldots]$ remercier très chaleureusement Lise McNicoll pour le travail remarquable qu'elle a accompli au cours de sa longue carrière à l'Institut. [...] Tous ceux qui l'ont connue peuvent témoigner qu'elle s'est dévouée de manière exemplaire pour l'Institut. [...] Lise McNicoll était appréciée de tous et elle représente toujours la mémoire vivante de l'IHAF (Alain Beaulieu, 2011).

Ce texte hommage a été rédigé par Guy Laperrière. L’ont signé les présidentes et présidents de l'IHAF et directrices et directeurs de la RHAF que nous avons pu rejoindre et qui se sont succédé de 1978 à 2010 :

\begin{tabular}{|l|l|}
\hline $\begin{array}{l}\text { Présidents, présidentes } \\
\text { de l'IHAF }\end{array}$ & Directeurs, directrices de la RHAF \\
\hline & JACQUES MATHIEU, 1977-1979 \\
\hline JACQUES MATHIEU, 1982-1983 & ANDRÉE DÉSILETS, 1982-1985 \\
\hline ReNÉ HARDY, 1983-1985 & JOHN DICKINSON, DIR. SC., 1982-1986 \\
\hline ANDRÉE DÉSILETS, 1985-1989 & RICHARD JONES, 1985-1988 \\
\hline JeAN ROY, 1989-1995 & RENÉ HARDY, 1992-1993 \\
\hline MICHELINE DUMONT, 1995-1997 & JACQUES ROUILLARD, 1993-1996 \\
\hline JOANNE BURGESS, 1997-1999 & FERNANDE ROY, 1996-2000 \\
\hline GUY LAPERRIÈRE, 1999-2001 & PIERRE LANTHIER, 2000-2003 \\
\hline CHRISTIAN DESSURAULT, 2001-2005 & DENYSE BAILLARGEON, 2003-2004 \\
\hline MARC VALLİERE, 2005-2009 & CHRISTINE HUDON, 2004-2007 \\
\hline ALAIN BEAULIEU, 2009-2013 & ROBERT GAGNON, 2007-2012 \\
\hline
\end{tabular}

